\title{
Bernardo O’Higgins y el patrimonio del vino en Chile
}

\author{
Bernardo O'Higgins and the wine heritage in Chile \\ Fernando Mujica1, Michelle Lacoste Adunka², Pablo Lacoste ${ }^{3 *}$
}

\section{RESUMEN}

Se examina el lazo entre el libertador de Chile, Bernardo O'Higgins, y la cultura de la vid y el vino. Se observa que, además de político, militar y patriota, fue un viticultor y amante del vino. O'Higgins cultivó la vid en sus haciendas de Chile (Las Canteras) y Perú (Cuiba). Se preocupó por elaborar vinos escogidos, como el asoleado. Además, el vino formaba parte de su dieta cotidiana. Valoraba los vinos de calidad y los utilizaba para homenajear a sus visitantes, sobre todo militares y estadistas. En este sentido, O’Higgins fue viticultor y, a la vez, contribuyó al fortalecimiento de la cultura de la apreciación del vino en Chile.

Palabras clave: patrimonio enológico, vinos patrimoniales, viticultura tradicional, cultura de la apreciación del vino.

\begin{abstract}
This paper examines the link between the liberator of Chile, Bernardo O'Higgins, and the culture of the vine and wine in this country. It is detected that, besides political and militar activity, he was a viticulturist and wine lover. O'Higgins cultivated the vine on his haciendas in Chile (Las Canteras) and Peru (Cuiba). He was concerned with making selected wines, such as the sunny one. In addition, wine was part of their daily diet. He valued quality wines, and used them to pay homage to his visitors, especially military men and statesmen. In this sense, O'Higgins was a wine grower and, at the same time, contributed to the strengthening of the wine appreciation culture in Chile.
\end{abstract}

Keywords: oenological heritage, heritage wines, traditional viticulture, wine appreciation culture.

\section{Introducción}

El papel de los grandes líderes, reyes, papas y emperadores en la valoración, visibilización y patrimonialización de los vinos es un elemento clásico en la historia de la vitivinicultura mundial. Como el valor del vino es subjetivo, y depende de un largo proceso de construcción cultural, los países europeos se han caracterizado, precisamente, por destacar el papel de los líderes en la historia de sus vinos. En la historia de los vinos de Jerez se resalta el papel que les cupo a reyes como Alfonso X y Felipe II (González Gordón, 1970). El reconocimiento del marqués de Pombal (1756) es recurrentemente destacado en la historia del Oporto (Ribeiro de Almeida, 2010). También se reconoce el papel del zar de Rusia, Pedro el Grande, en la difusión del vino de Cahors (Nérand, 2016), lo mismo que el papa Juan XXII y el rey Francisco I de Francia (Lacoste, 2016; Grizet y Laborie, 2016).

La historia del Tokay resulta esclarecedora. Este vino generoso se ha presentado como el mejor vino dulce del mundo. Su característica cualitativa descansa sobre el clima de Hungría, donde crece la vid y se desarrolla la Botrytis cinarea, hongo que produce la podredumbre noble de las uvas. Sin embargo, el valor simbólico del Tokay radica en su historia. Fue el mejor vino del Imperio Habsburgo, sus viñedos estaban en manos del emperador. También fue el vino favorito de Francisco Rákóczi II, líder de la guerra de la independencia húngara. De igual manera, fue el mosto preferido de Federico I de Prusia y de los zares de San Petersburgo, como Pedro el Grande. La fama y el prestigio del Tokay fue tal, que el mismo Rey Sol, Luis XIV de Francia, lo denominó "le roi des vins et le vin des rois" (Johnson, 2005).

\footnotetext{
Escuela de Sommeliers. Santiago, Chile.

Universidad San Sebastián. Santiago, Chile.

Universidad de Santiago de Chile. Santiago, Chile.

Autor correspondiente: pablo.lacoste@usach.cl
} 
Producto cultural por excelencia, el vino es muy sensible al proceso de patrimonialización que le aportan las grandes figuras. Estas le dan atributos significativos, al asociarse con procesos históricos complejos y fortalecer las representaciones del vino, como parte de una construcción cultural colectiva, de larga duración y profundamente arraigada en el territorio.

Los vinos patrimoniales representan una rama significativa dentro de la vitivinicultura de Chile. Incluyen los vinos de Codpa y Pintatani (Castro y Mujica, 2015), pajarete (Ramírez, 2015; Castro et al., 2016), chacolí (Lacoste, et al., 2015), pipeño (Aguilera y Alvear, 2017) y asoleado de Cauquenes y Concepción (Lacoste et al., 2016), entre otros. El vino escogido más antiguo es el pajarete, registrado en Chile desde 1790. Le siguen en antigüedad el chacolí y el asoleado, que hicieron su aparición en los banquetes oficiales de 1817. Más tarde se registraron los vinos de Codpa y el pipeño. Además, el pajarete y el asoleado de Concepción y Cauquenes fueron los primeros vinos de América delimitados como Denominación de Origen por parte del Estado (1953). Con el pipeño se ha creado un trago muy popular, el "terremoto", de creciente interés cultural y patrimonial (Aguilera y Alvear, 2017).

Poco a poco, la historiografía ha comenzado a aportar luz sobre la historia de estos vinos. En ese marco, conviene examinar el papel que les cupo en este proceso a los patriotas y primeros estadistas. Ya se han detectado aportes importantes: José de San Martín, además de destacarse como militar y político, se dedicó a cultivar la vid, elaborar el vino, y apreciarlo en la vida social (Lacoste, 2013). Por su parte, Domingo Faustino Sarmiento se dedicó intensamente a impulsar la producción vitivinícola, tanto en Chile como en Argentina (Gironés, 2012).

El objetivo de este artículo es conocer una nueva dimensión de la vida de Bernardo O'Higgins, como viticultor y amante del vino. Se ha utilizado el método propio de la historia (heurístico crítico). Se han examinado principalmente las memorias, cartas y documentos producidos por los patriotas y sus colaboradores. Se incluyen las cartas de Bernardo O'Higgins y José de San Martín y las memorias de actores de la época.

La trayectoria militar y política de Bernardo O'Higgins (1778-1842) es conocida. Hijo del virrey del Perú, Ambrosio O’Higgins, Bernardo heredó un singular capital sociocultural. Se educó en Europa, donde conoció a Francisco Miranda, precursor de la independencia hispanoamericana. Regresó a su país en 1804 para administrar la estancia Las Canteras, heredada de su padre. Participó activamente en la Patria Vieja (1810-1814), primera experiencia de gobierno patrio en Chile. Tras la reconquista española, se exilió en Mendoza, mientras los realistas destruían su hacienda Las Canteras. Luego participó en el Ejército de los Andes, con el cual regresó a Chile y derrotó a los españoles en las batallas de Chacabuco (1817) y Maipú (1818). Ello le abrió el camino al gobierno de Chile, donde fue Director Supremo por seis años (1817-1823). Después de salir del poder, se radicó en Perú, donde vivió hasta su muerte (1823-1842) dedicado al cultivo de dos haciendas.

La historia de Chile ha dedicado grandes libros para estudiar la vida y la obra de O'Higgins, principalmente desde el punto de vista político y militar. Ahora conviene examinar la dimensión enológica de su vida.

\section{Viticultor en el valle del Itata (1804-1814)}

La tarea de Bernardo O'Higgins como viticultor comenzó en la hacienda Las Canteras, situada cerca de Chillán. Recibió esta propiedad como herencia de su padre, el virrey don Ambrosio O'Higgins, con 3.000 cabezas de ganado. Después de sus viajes por Perú y Europa, Bernardo O’Higgins regresó a Chile en 1804 y se dedicó a la administración de esta hacienda.

En Las Canteras se cultivaba la viña y se elaboraba el vino. Particularmente buena fue la cosecha de 1812. "Aquí me estoy regalando con el mosto, que está muy particular. Solamente habiéndolo visto, pudiera haber creído fuese de Canteras" (...) "Son tan buenos estos mostos, que no puedo creer sean de Las Canteras", le escribió O'Higgins a Isabel Riquelme el 31 de mayo de aquel año (O’Higgins, 2011).

La hacienda Las Canteras estuvo en el centro del escenario militar de las guerras de la independencia. Para abastecer al ejército patriota, O'Higgins donó 10.000 cabezas de ganado que tenía en esta propiedad, durante el sitio de Chillán (1813). Después de la reconquista española, los realistas tomaron duras represalias por estas acciones y destruyeron totalmente la hacienda de O'Higgins. "Las tierras dejadas por mi padre, extensas y fértiles, habían sido reducidas a un desierto por los soldados españoles y sus partidarios. Desde entonces han 
quedado totalmente improductivas" (O'Higgins, 2011). El patriota se lamentó de esta pérdida, sobre todo por sus viñas: "Quemaron mi magnífica casa y destruyeron mis viñas" (O'Higgins, 2011). Con la caída de la Patria Vieja se cerró la etapa chilena del O'Higgins-viticultor.

La actividad de O'Higgins como viticultor se desenvolvió en el marco del paradigma tradicional, hispano-criollo. El vino se elaboraba principalmente a partir de la variedad Listán Prieto, llamada después "uva País" en Chile y "criolla chica" en Argentina; o bien, uva negra corriente en Perú y uva misión en California. Las viñas eran unidades pequeñas, de entre 1.000 y 2.000 plantas. Se abonaban con estiércol de gallina o de oveja y no se usaban productos químicos. Además, la cosecha se realizaba en forma manual. Después de la vendimia, la uva se pisaba con pie de hombre, o en forma manual, utilizando zarandas de colihues. El mosto se fermentaba en tinajas de greda. Recipientes parecidos se usaban después en la bodega para criar, conservar y añejar los vinos. El método era totalmente artesanal. Dentro de este paradigma se desarrollaron los demás vinos patrimoniales de Chile: pipeño, chacolí, asoleado y pajarete, entre otros.

\section{Vinos patrimoniales en banquetes de la Patria (1811-1817)}

Los banquetes de la Patria fueron las celebraciones organizadas por el gobierno de Chile para conmemorar la gesta emancipadora, cohesionar al grupo dirigente y atraer a los hacendados para sostener el esfuerzo económico que requerían las campañas militares en marcha. Entre los banquetes de la Patria se destacan principalmente dos eventos: la celebración del primer aniversario de la toma del poder por parte de los patriotas (30 de septiembre de 1811) y el festejo de la batalla de Chacabuco y homenaje al primer Director Supremo de Chile, el día de su asunción (16 de febrero de 1817).

Estos eventos se realizaron en los salones más elegantes disponibles y contaron con la presencia de las más distinguidas damas y caballeros de la causa independentista. La fiesta del 30 de septiembre de 1811 se hizo en el Palacio de la Moneda. Y la de 1817 fue en casa de José Enrique Rosales, quien vivía en calle Compañía 126 (Zapiola, 1840), en la ciudad de Santiago. Se contrataron músicos para animar las veladas y se organizaron bailes.
Los vinos estuvieron presentes en ambas fiestas. En la celebración de 1811 se sirvieron "los mejores vinos del país y los peninsulares se escanciarían en la más fina cristalería, y relumbrarían la plata y la vajilla de porcelana de la China" (Vidal, 2010). Ese fue el tímido estreno de los vinos en un evento de esta naturaleza, organizado por los Carrera, entonces en el cenit del poder.

Mayor contenido patrimonial tuvieron los vinos del banquete del 16 de febrero de 1817. Recordemos que fue organizado por O'Higgins, el mismo día de su asunción del mando de Director Supremo de Chile. En esta celebración se sirvieron específicamente los vinos patrimoniales chilenos, incluyendo "chacolí de Santiago, asoleado de Concepción y no pocos vinos peninsulares" (Pérez Rosales, 1882; Rojas, 1940).

Hubo una decisión desde la cabeza del Estado, de visibilizar y patrimonializar los vinos típicos chilenos. Por primera vez llegaban al principal escenario político nacional, en la mesa presidencial. Fueron colocados allí por la mano del patriota.

\section{Viticultor en Perú (1824-1842)}

Para sostener su vida en Perú, O’Higgins tenía dos haciendas: Montalván y Cuiba. Ambas le fueron donadas por el entonces Protector del Perú, su amigo José de San Martín, en compensación por los servicios prestados a la causa de la independencia. La decisión fue dejada en firme, poco después, por decreto del 30 de marzo de 1822 del gobierno peruano. Y fueron el recurso que le quedó a O'Higgins después de su derrocamiento. "No puedo contar con otros fondos que Montalván y Cuiba, con que la generosidad del gobierno del Perú y de mi mejor amigo habían recompensado mis servicios", le escribió a San Martín el 5 de marzo de 1823 (O'Higgins, 2011). Con esta expectativa partió el libertador de Chile hacia su exilio en Perú. Grande fue su sorpresa al llegar, pues comprobó que ambas propiedades estaban en pésimas condiciones. "Montalván y Cuiba han sido completamente arruinadas por el enemigo", escribió el 9 de agosto de 1823 (O'Higgins, 2011). Se propuso entonces trabajarlas intensamente, para ponerlas de nuevo en marcha.

Ambas haciendas se hallaban en el Valle de Cañete, 150 kilómetros al sur de Lima. Montalván estaba a tres leguas del puerto de Cerro Azul, el cual fue habilitado para el cabotaje en el siglo XIX. La conexión con Lima se realizaba de manera eficiente, con sistema de transporte multimodal: 
el primer tramo era por camino de herradura $(15 \mathrm{~km})$ entre las haciendas y el puerto y lo hacían los arrieros; el segundo era por mar (145 km), entre los puertos de Cerro Azul y El Callao; y el tercero era el camino carretero de El Callao a Lima. La producción principal de Montalván era el cultivo de la caña, para elaborar azúcar y ron. En cambio, la hacienda de Cuiba sí se dedicó al cultivo de la vid y la elaboración del vino. En esta propiedad, precisamente, el libertador pudo retomar su antiguo oficio de viticultor.

La vida de O'Higgins en Perú transcurrió entre sus haciendas y su casa de Lima. Al principio, el libertador se instaló en las propiedades rurales para ponerlas nuevamente en producción. Posteriormente, delegó la administración en Pedro Aldunate y José Toribio Pequeño y otros colaboradores, para permanecer más tiempo en Lima y atender asuntos personales, políticos y diplomáticos. La correspondencia mantenida con Aldunate y Pequeño ha permitido conocer sus prácticas de consumo y su cultura de la apreciación del vino.

"Celebro que los potreros y viñas de Cuiba y se hayan limpiado y podádose las últimas. Del parrón grande, cuya poda es en la menguante del presente mes, podrá sacarse alguna planta, de la que no sea quebrantada, para el replante de las dos viñas, que sin duda se habrá perdido alguna; el parrón (de la) huerta, también da para sacar alguna planta", le escribió a José Toribio Pequeño el 24 de agosto de 1841 (O’Higgins, 2011).

Los vinos de O'Higgins no eran comunes, sino escogidos. Inspirado en la tradición de los vinos de mejor calidad de Cauquenes y Concepción, el patriota se decantó por elaborar vinos asoleados. Tal como hacían los viticultores surmaulinos, en la hacienda Cuiba se procedía a exponer la uva al sol, después de la cosecha, para lograr la deshidratación y la concentración del azúcar. El 25 de marzo de 1840, el libertador le escribió al administrador:

"Mi presente estado de salud no me permite el gusto de ir a la vendimia de Cuiba. Mañana escribiré a don J.A. Sepúlveda para que acompañe a V. el día de la pisa porque hacemos nuestro vino sin cocido, todo lo que se necesita que la uva tenga al menos cuatro días de cortada y tendida al sol antes de su beneficio. Lorencito y Santander han visto muchas veces como esto se hace" (O’Higgins, 2011).

Los vinos de la hacienda Cuiba marcaban una continuidad con los mejores vinos que O'Higgins había degustado en su juventud, entre Talca y Chillán, y los que se habían servido en el banquete de la Patria, el día de su asunción al poder, el 16 de febrero de 1817.

Junto con los vinos asoleados, la hacienda también tenía una línea de vinos fortificados con alcohol. Esta era una práctica habitual en la región para prevenir el deterioro del producto, sobre todo cuando debían realizarse largos viajes. Estas costumbres regían también en Europa, para vinos de exportación, como el Oporto del norte de Portugal. De todos modos, a veces sucedía que los viticultores se excedían en las proporciones, y los vinos quedaban con excesiva graduación alcohólica. El libertador estaba atento a estos detalles, y se ocupaba de buscar soluciones. Así se infiere de la carta que le escribió a José Toribio Pequeño el 8 de septiembre de 1841:

"Cuando V. tenga tiempo de mandarme dos barriles de vino nuevo, me los remitirá V. Me servirá dicho vino para mezclarlo con otros dos barriles del añejo que vinieron y está demasiado fuerte por el licor con que estaban mezclados. Así ahorraré \$20 mensuales, a lo menos, que cuesta el que se compra” (O’Higgins, 2011).

El documento es interesante, porque muestra en detalle las prácticas enológicas del patriota. Además revela que el vino formaba parte de su dieta cotidiana y su estilo de vida.

Los vinos de O'Higgins se guardaban en la hacienda y desde allí se remitían a Lima, cada vez que era necesario, para el consumo del libertador y sus amistades. Se produjo así un sostenido tráfico de barriles de vino, que llevaban los arrieros desde Cuiba y Montalván hasta Lima. $\mathrm{Y}$ en la correspondencia de O'Higgins con su administrador, Pedro Aldunate, se entregan detalles de interés. "Los barriles que lleva Padilla son 24, y aquí no quedan más que 13 por todos, inclusos los de vino", le escribió el 23 de enero de 1830 a Aldunate (O'Higgins, 2011). Poco después, el 1 de mayo, escribió: "Hemos recibido los dos barriles de vino, que probaremos en cuanto se repose un poco" (O’Higgins, 2011). 
La constante mención del tema en la correspondencia de O'Higgins con los administradores de sus haciendas revela su interés por el vino. Para el patriota, el vino era mucho más que un producto del campo. Formaba parte de su vida cotidiana, tanto en su alimentación como en su vida de relación.

\section{El patriota y la cultura de la apreciación del vino}

La calidad de los vinos era un tema de interés para el libertador. En algunos casos, se solicitaba controlar bien la calidad de los vinos antes de enviarlos: "Si hay un barril de vino que esté bueno, y no picado, me lo mande por la recua que traiga la azúcar", le escribió a Aldunate el 17 de abril de 1830 (O'Higgins, 2011). La calidad de los vinos era un tema de interés constante para el libertador. Así lo expresó en otra carta a Aldunate, fechada el 23 de abril de 1831: "Los vinos están escasos y muy malos; nos vendrían bien un par de barriles" (O’Higgins, 2011).

Junto con los vinos peruanos de su hacienda, O'Higgins valoraba también los vinos chilenos. Estos no eran abundantes en Perú, pero cada tanto, algún amigo le abastecía con los caldos patrios. En 1830, el libertador recibió los vinos de Talcahuano, que le trajo Cayetano Parrasí. El patriota resolvió remitirlos a su hacienda, para conservarlos en lugar seguro. En carta del 7 de julio informó de la llegada de Parrasí desde Talcahuano con preciados alimentos. Algunos los remitió a Montalván y otros los retuvo en Lima, hasta poder enviarlos con mayor seguridad. "Quedan aquí tres barriles de vino que también ha entregado Parrasí y no van con la presente recua porque los burros, según el arriero, no pueden llevarlos por su peso, pero en primera oportunidad de mulas marcharán" (O’Higgins, 2011).

Para O'Higgins, el vino chileno era un elemento precioso, y solo se podía enviar a largas distancias a través de personas de confianza. El servicio regular de transporte terrestre no era adecuado para arriesgar este patrimonio. "No lleva don Tomás los tres barriles de vino por falta de mulas y no fiarlos de arrieros que, para aliviar el peso, saquen por medios que no les son desconocidos, una parte del vino", le escribió el 16 de julio de 1830 a Aldunate (O’Higgins, 2011).

La cultura de la apreciación del vino formó parte de la vida del libertador. Además de degustar los vinos chilenos y peruanos, se interesaba por los vinos europeos, particularmente, para agasajar a sus visitantes de relevancia. "Si viniese el ingeniero don Tomás Gil -escribió el 28 de febrero de 1838- sería necesario mandar uno o dos cajoncitos de vino Bordeaux. Zapata los suele tener de doce botellas cada uno y aunque vende caro, pero casi siempre bueno" (O’Higgins, 2011). Más adelante, con motivo de la presencia en Perú del general Bulnes, por la guerra con la Confederación Peruano-Boliviana, el prócer lo invitó a su hacienda de Montalván y, para atenderlo, ordenó vinos europeos. Para ello solicitó acudir a un barco surto en el Callao, con el fin de comprarle "algunas botellas de cerveza o de vinos europeos que nos podrían servir para cuando vaya el general Bulnes" (O’Higgins, 2011).

La actitud del patriota frente al consumo de vinos era flexible y se adaptaba a las circunstancias. Consciente del avance del paradigma francés, aceptaba incorporar en su mesa los vinos de Burdeos para homenajear a figuras emblemáticas. Paralelamente, en su viña, elaboraba los vinos con el estilo tradicional chileno, con Listán Prieto Uvas Paí y las técnicas del asoleado de Cauquenes. En todo caso, el vino era parte indispensable en su mesa.

\section{Conclusión}

Esta investigación ha permitido observar una cara hasta ahora desconocida del libertador Bernardo O'Higgins: su carácter de viticultor y su interés por la cultura de la apreciación del vino. El patriota se dedicó a cultivar la viña y elaborar el vino, tanto en Chile como en Perú. Y prestó atención especial a esta bebida, tanto en su mesa cotidiana, como en los banquetes públicos y en los homenajes a invitados importantes.

El patriota llegó a conocer el paradigma francés y los vinos de Burdeos, sobre todo, durante su estadía en Perú. Tuvo oportunidad de comparar con los vinos tradicionales, del paradigma hispano criollo. Como resultado, optó por otorgar a cada vino una función específica: los vinos de Burdeos servirían para la vida social, principalmente para engalanar la mesa en homenaje a personajes del poder. En cambio, para su consumo particular, prefirió mantenerse dentro de los vinos tradicionales, elaborados a partir de uva Listán Prieto y variedades criollas. Dentro de este paradigma, O'Higgins se esmeró por obtener los vinos de la mejor calidad posible, siguiendo las técnicas que los viticultores surmaulinos habían desarrollado para los vinos asoleados. El patriota 
elaboró sus propios asoleados en la hacienda de Cuiba, en el valle de Cañete. Y se preocupó por cuidar la calidad de los vinos.

De todos modos, la cultura de la apreciación del vino chileno la mantuvo durante su estadía en el Perú. Así lo revela el cuidado que dedicaba a los vinos que le llegaban desde el puerto de Talcahuano. En sus cartas demostró este interés, lo mismo que por otros productos gastronómicos de su tierra, como el queso de Chanco y el jamón de Chiloé. En cierta forma, O'Higgins se mantuvo dentro del canon gastronómico que él mismo estableció en los banquetes de la Patria del 16 de febrero de 1817.

Después de la muerte de O'Higgins, la situación cambió, con la imposición del paradigma francés dentro de la viticultura regional y mundial. Las principales viñas chilenas se subordinaron a ese paradigma, y abandonaron los vinos patrimoniales, del modelo anterior. Las variedades hispanocriollas, como Listán Prieto Uva País, fueron denostadas y minimizadas por las nuevas tendencias. Durante un siglo y medio la hegemonía del paradigma francés dejó muy poco espacio para la valoración de los vinos patrimoniales.

De todos modos, en los últimos años ha comenzado lentamente a ponerse en cuestión la cultura enológica dominante, con la revaloración de la viticultura tradicional y su cepa emblemática, la uva País. Con ellos, se percibe también una recuperación de los vinos patrimoniales, aquellos que valorizó el libertador Bernardo O’Higgins.

\section{Agradecimiento}

Los autores agradecen al Proyecto FONDECYT 1130096 por el financiamiento otorgado.

\section{Literatura citada}

Aguilera, I.; Alvear, A.

2017. Pipeño y Terremoto como bebidas nacionales: una reflexión en torno a la patrimonialización y la representación de la nación. RIVAR, 4 (12): 7-22.

Castro, A.; Pszczólkowski, Ph.; Mujica, F.; Lacoste, P.; Núñez,

E.; Cofré, C.; Lacoste Adunka, M.; Soto, N.

2016. El pajarete de Huasco y Elqui (Chile). Historia de un vino escogido. Idesia, 34 (4): 23-35.

Castro, A.; Mujica, F.; Argandoña, F.

2015. Entre Pintatani y Codpa. Paisaje y productos típicos en los relatos campesinos, 1847-2013. RIVAR, 2 (6): 70-86.

Gironés de Sánchez, I.

2012. Domingo F. Sarmiento y la iniciación de la gran vitivinicultura industrial argentina. Edición Homenaje. San Juan, Argentina. 382 p.

González, F.; Chiappero, M.L.

2017. El banquete y la identidad noble. Su representación en la literatura de la Baja Edad Media (s. XIV). RIVAR, 4 (11): 74-91.

González Gordon, M.

1970. Jerez-Xerez Sherish. Noticias sobre el origen de esta ciudad, su historia y su vino. Gráfica Domingo. Jerez, España. 610 p.

Griset, P.; Laborie, L.

2016. Historiographie et réinvention du vignoble de Cahors, XXe-XXIe siècles. RIVAR, 3 (7): 81-102.

Johnson, $\mathrm{H}$.

2005. Historia del vino. Editorial Blume. Barcelona, España. $256 \mathrm{p}$.

Nérard, F.-X.

2016. Du Cahors au kagor. Pistes pour une histoire du vin de Cahors en Russie. RIVAR, 3 (7): 25-39.
Lacoste, P.; Castro, A.; Briones, F.; Cussen, F.; Soto, N.; Rendón, B.; Mujica, F.; Aguilera, P.; Cofré, C.; Núñez, E.; Michelle Lacoste Adunka, M.

2015. Vinos típicos de Chile: ascenso y declinación del Chacolí (1810-2015). Idesia, 33(3): 97-108.

Lacoste, P.; Castro, A.; Rendón, B.; Pszczólkowski, Ph.; Soto, N.; Lacoste Adunka, M.; Jeffs, J.; Salas, A.; Cofré, C.; Núñez, E.

2016. Asoleado de Cauquenes y Concepción: apogeo y decadencia de un vino chileno con Denominación de Origen. Idesia, 34 (1): 85-99.

O'Higgins, B.

2011. Cartas de Bernardo O’Higgins. Editadas por Cristian Guerrero Lira y Nancy Miño. Editorial Historia Crítica. Santiago, Chile. 320 p.

Pérez Rosales, D.

1882. Recuerdos del pasado (1814-1860). Editorial Jackson, 1946. Buenos Aires, Argentina. 533 p.

Ramírez, S.; Ramírez, C.

2015. El Pajarete. En Aravena, R. (editor). Patrimonio vitivinícola: aproximaciones a la cultura del vino en Chile. Ediciones Biblioteca Nacional. Santiago, Chile. pp. 82-89.

Ribeiro de Almeida, A.

2010. A Autonomia Jurídica da Denominacao de Origem. Uma perspectiva transnacional. Uma grarantia de qualidade. Coimbra, Wolters Kluwer. Coimbra, Portugal, 1475 p.

Rojas, R.

1940. El santo de la espada. Vida de San Martín. Losada. Buenos Aires, Argentina. 526 p.

Vidal, V.

2010. Javiera Carrera, madre de la Patria. Editorial RIL. Santiago, Chile. 242 p.

Zapiola, J.

1871. Recuerdos de treinta años (1810-1840). Editorial Zigzag, 1945. Santiago, Chile. 311 p. 